\title{
Redes definidas por software: Solución para servicios portadores del Ecuador
}

\author{
Gabriela Cuenca Pérez / Mónica Flores Marín
}

\section{Resumen}

Actualmente las Redes Definidas por Software (SDN) se muestran como una arquitectura vanguardista que ha cambiado el modelo de administración de redes por un concepto más programable y centralizado sin necesidad de aumentar el hardware, para así cumplir con las exigencias del mundo del internet de buscar redes más eficientes. Este artículo tiene como propósito exponer sobre los servicios portadores y la evolución de SDN, explicándola desde sus inicios hasta las aplicaciones que existen en la actualidad, seguido de su arquitectura y componentes. Se concluye que SDN es una posible solución para los servicios portadores del Ecuador, ya que la red es programable y permite dar paso al uso de políticas y reglas para que sea más eficiente con respecto a los costos operacionales. Adicionalmente se presenta como una nueva tecnología, donde se espera que exista un mayor respaldo en la seguridad del sistema estableciendo estándares de protección contra delito informático.

\section{Palabras clave:}

Redes Definidas por Software, Servicios Portadores, Seguridad.

\begin{abstract}
Nowadays Software Defined Networking (SDN) is shown as a modern architecture that has changed the administration network model. This paper aims to describe carriers and the evolution of SDN, explaining it from its beginnings to the applications that exist today, followed by its architecture and components. We conclude that SDN is a possible solution for the carrier services of Ecuador, since the network is programmable and also can also make way for using policies and rules to make it more efficient with respect of operational costs. In addition to that, it is presented as a new technology, which is expected to be a greater support in establishing safety standards for protection against cybercrime.
\end{abstract}

\section{Keywords:}

Software Defined Networking, Carriers, Security. 


\section{Introducción}

Las redes de Telecomunicaciones, con el pasar de los años han ido incrementando; se estima que 2,937 millones de usuarios (Internet World Stats, 2013) usan internet como vía de acceso a diversos servicios. El $40 \%$ de la población mundial tiene una conexión de internet (Internet Live Stats, 2014). Según Cisco, el tráfico global de datos móviles de Internet creció casi 18 veces desde el 2002 hasta el 2012 (Cisco Systems Inc, 2014).

El incremento del tráfico de Internet causado por el uso de servicios como almacenamiento en la nube, telefonía IPI, video, virtualización (Ortiz Jr., 2013), requiere que no sólo los proveedores de telecomunicaciones, sino también organizaciones estatales, universidades, centros de salud, empresas y data centers busquen soluciones que se adapten y refuercen las redes tradicionales (Parol \& Pawlowski, 2013). Según Sezer et al. (2013), la configuración e instalación de redes de telecomunicaciones implica adquirir personal altamente calificado y más equipos de transmisión (Routers, Firewalls, Switches, etc.) y aumentar costos operacionales.

De acuerdo a Feamster, N., Rexford, J., y Zegura, E. (2014), los routers y switches manejan software de distribución controlada y compleja que normalmente son software propietario y de código cerrado, donde se implementa protocolos de red que pasan por años de estandarización y pruebas de interoperabilidad. Los administradores de red configuran típicamente los dispositivos de redes individuales utilizando interfaces de configuración, que varían entre los vendedores e incluso a través de diferentes productos del mismo proveedor. Aunque algunas herramientas de gestión de red ofrecen un punto de vista central para la configuración de esta, estos sistemas aún continúan operando al nivel de protocolos,

1 Protocolo de internet mecanismos e interfaces de configuración. Es por esto que el modo de operación ha ido frenando la innovación, incrementado la dificultad e inflando, tanto el capital como los costos operativos de administración de red.

Actualmente en el sector de Telecomunicaciones, existe una nueva arquitectura que ha estado cambiando el modelo de las redes (CITRIX, 2014; Kobayashi et al., 2014; Shin, Nam, \& Kim, 2012; Feng, Bi, \& Wang, 2014). Esta nueva tecnologia se llama red definida por software o SDN por sus siglas en inglés, y permite a los usuarios añadir velocidad, características mediante la adquisición de software y dispositivos de redes de bajo costo que mueven el tráfico de Internet (Burrows, 2014; Open Network Foundation, 2012). Según la Unión Internacional de Telecomunicaciones (2014) el SDN se considera un cambio importante en la tecnología de red el cual les dará a los operadores de telecomunicaciones, la capacidad de establecer y gestionar nuevos recursos de redes sin desplegar nuevas tecnologías.

En el Ecuador, dada la importancia de crear sistemas económicos, eficientes y seguros, el gobierno, de acuerdo a la Ley Especial Telecomunicaciones Reformada 184 (2011), exige a los portadores que cumplan con lo requerido. Los servicios portadores del Ecuador no han implementado un sistema SDN de administración eficiente que permita una solución para la optimización de redes (Vivar, 2014; Montero, 2014; Yépez, 2014).

El objetivo del presente trabajo es presentar una alternativa de solución de redes para los servicios portadores del Ecuador, partiendo del estado de crecimiento de los portadores, seguido de la evolución de SDN y de su arquitectura y componentes. Además se demuestra las implementaciones de estrategias que en la actualidad se están utilizando en las grandes compañías de redes (Darbha, Shevenell, \& Normandin, 2013; Douligeris \& Serpanos, 2007; Gupta, 2013; 
Metzler, 2012; ONF, 2013; Raza, Sivakumar, Nafarieh, \& Robertson, 2014; Scott-Hayward, O'Callaghan, \& Sezer, 2013; Sezer, y otros, 2013; Shackleford, 2013). Así mismo se realizará entrevistas en empresas de telecomunicaciones del Ecuador, de las cuales una es vendedora internacional de tecnología en base a SDN y las otras dos son empresas que proporcionan servicio de portadores en el Ecuador para demostrar el estado actual del país con respecto a la nueva tecnología.

\section{Marco teórico}

En el desarrollo de este documento se presenta, la situación actual del Ecuador con respecto a sus portadores, seguida de la evolución de SDN, su arquitectura y componentes, junto a las estrategias de implementación y seguridades de SDN.

\section{Situación actual del Ecuador}

Dentro de la Ley Especial de Telecomunicaciones Reformada en su capítulo III, artículo 24 declara que: "Plan de Desarrollo (Sustituido inc. 2 por el Art. 7 de la Ley 94, R.O. 770, 30-VIII-95).- El Plan de Desarrollo de las Telecomunicaciones tiene por finalidad dotar al país de un sistema de telecomunicaciones capaz de satisfacer las necesidades de desarrollo, para establecer sistemas de comunicaciones eficientes, económicas y seguras". El Estado debe asegurar que la infraestructura para conectividad y telecomunicaciones cubra todo el territorio nacional, de modo que las Tecnologías de la Información y Comunicación (TIC'S) estén al alcance de toda la sociedad de manera equitativa (Llerena \& Ruiz, 2013). Además la Encuesta Nacional de Empleo Desempleo y Subempleo (ENEMDU) (2011 - 2013), indica que el $40,4 \%$ de la población ecuatoriana ha utilizado Internet en el año 2013, 11,4 puntos más que lo registrado en el 2010, lo cual denota un mayor incremento de la inclusión digital en el pueblo ecuatoriano y mayor demanda al acceso a Internet.

\section{Servicios Portadores}

Los servicios portadores han tenido un incremento muy notorio en los últimos años con respecto a su número de usuarios; además se está convirtiendo en uno de los servicios con más ingresos económicos según reportes de la Superintendencia de Telecomunicaciones (SUPERTEL) (Carrillo, León, Ramos \& Yépez, 2009), los servicios portadores proporcionan al usuario la capacidad necesaria para el transporte de información, independientemente de su contenido y aplicación, entre dos o más puntos de una red de telecomunicaciones y se pueden prestar bajo dos modalidades: redes conmutadas y no conmutadas (SUPERTEL, 2010). En la tabla 1 se muestra los siete primeros portadores del Ecuador.

\section{Tabla \# 1}

\section{Servicio Portadores del Ecuador}

\begin{tabular}{|l|cc|}
\hline \multicolumn{1}{|c|}{ Operadora } & Número de Usuarios & Número de Enlaces \\
\hline CNT EP. & 671.717 & 718.764 \\
SURATEL SA. & 168.399 & 178.302 \\
ECUADORTELECOM S.A. & 117.298 & 121.940 \\
MEGADATOS S.A. & 50.876 & 53.083 \\
PUNTONET S.A. & 23.008 & 27.231 \\
UNIVISA S.A. & 17569 & 17569 \\
TELCONET S.A. & 3672 & 25547 \\
\hline Nota: Adaptado de "Distribución del mercado de servicios portadores (Usuarios-AGO-14)", por SUPERTEL, 2014. Estadisticas Portadores.
\end{tabular}


En la figura 1 se observa como los servicios portadores del Ecuador presentan un crecimiento significante en el número de enlaces registrados desde el 2011 al 2014, en donde la Corporación Nacional de Telecomunicaciones (CNT) presenta un incremento mayor que los demás servicios portadores. Según Tomkins (2014) el crecimiento de los servicios portadores se debe al impul- so del servicio de computación en la nube, y al aumento de demanda, entre otros.

El objetivo de los servicios portadores es reducir los costos en general (Barkai, 2013; Davy, 2011) y las redes definidas por software tiene el potencial de minimizar gastos operacionales a través de la automatización y optimización de la red.

\section{Figura 1.}

Crecimiento del número de los enlaces registrado de los Servicios Portadores del Ecuador correspondiente al período del 2011 al 2014. Adaptado de "Servicios Portadores", por Supertel, 2014.

\section{Crecimiento de Servicios Portadores}

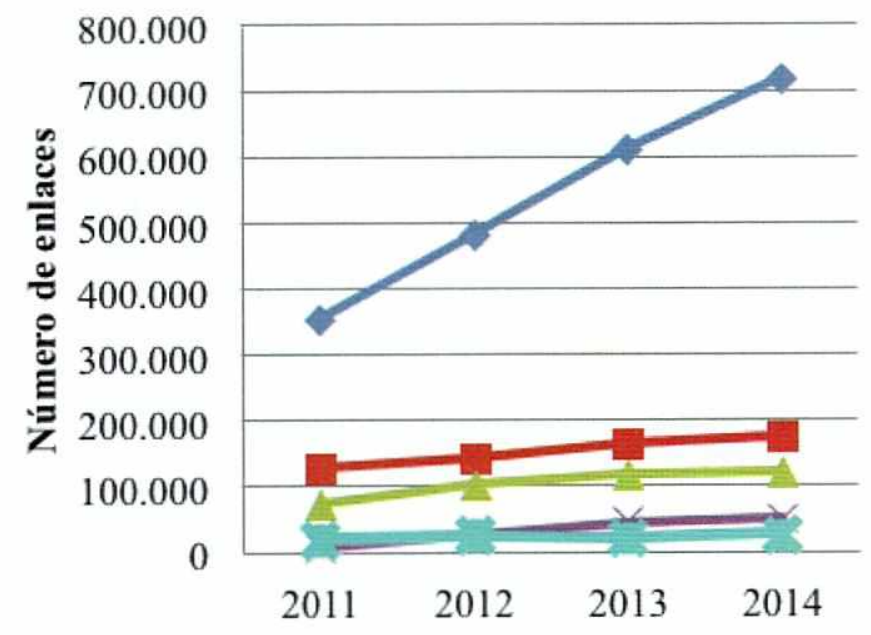

$\leadsto$ CNT EP.

$=$ SURATEL SA.

ECUADORTELECOM S.A.

$\because$ MEGADATOS S.A.

TELCONET S.A.

Fuente: Elaboración propià

\section{Evolución de SDN}

El término red definida por software ha ido evolucionando a lo largo del tiempo (Sezer et al., 2013), SDN ha estado desde 1996 tomando los nombres que aparecen en la tabla 2, en la cual las implementaciones de investigación e industrias incluyen desde GSMP de Ipsilon hasta OpenFlow. (Crabbe, 2011). 


\section{Tabla \# 2}

\begin{tabular}{|c|c|c|c|}
\hline \multicolumn{4}{|c|}{ La evolución de SDN } \\
\hline Nombre & Implementación & Аก̃o & Descripción \\
\hline GSMP & Ipsilon & 1996 & $\begin{array}{l}\text { Protocolo de administración de } \\
\text { Switches (General Switches } \\
\text { Managment Protocol) }\end{array}$ \\
\hline La tempestad & $\begin{array}{l}\text { Universidad de } \\
\text { Cambridge }\end{array}$ & 1998 & $\begin{array}{l}\text { Un sistema para guardar, salvar } \\
\text { recursos, redes programables }\end{array}$ \\
\hline ForCES & IETF & 2000 & $\begin{array}{l}\text { Reenvío y control de los elementos } \\
\text { de separación (Forwarding and } \\
\text { Control Element Separation) }\end{array}$ \\
\hline PCE & IETF & 2004 & Path Computation Element \\
\hline Ethane & $\begin{array}{l}\text { Universidad de } \\
\text { Stanford }\end{array}$ & 2007 & \\
\hline OpenFlow & $\begin{array}{l}\text { Open } \\
\text { Networking } \\
\text { Foundation }\end{array}$ & 2008 & \\
\hline
\end{tabular}

Nota: Adaptado de "A long road to SDN", por E. Crabbe, 2011, About Tech Field Day.

GSMP o General Switch Management Protocol, es un protocolo de propósito general para controlar un switch $\mathrm{ATM}^{2}$. GSMP permite a un controlador establecer y liberar conexiones a través de un switch. Además agrega y elimina nodos en una conexión de punto a multipunto; así como también maneja puertos de switch, solicita información de configuración y solicita estadísticas, también permite que el switch informe al controlador de eventos asíncronos. GSMP se ejecuta a través de un enlace ATM conectando el controlador al switch, en una conexión de control (canal virtual) establecida en la inicialización. El protocolo GSMP es asimétrico, siendo el controlador el master y el switch el esclavo donde múltiples switches pueden ser controlados por un solo controlador utilizando múltiples instancias del protocolo sobre las conexiones de control independiente (Newman et al., 1996).

Posteriormente se crea el framework La Tempestad; este provee un ambiente de red programable permitiendo la introducción y modificación dinámica de los servicios de red en dos niveles de granularidad. Primero, los switchlets 3 y los conceptos asociados de redes virtuales permiten la introducción de una arquitectura alternativa de control 
dentro de una red operacional. Segundo, el framework La Tempestad permite el refinamiento de servicios en un nivel más detallado de granularidad por medio del concepto de cierre de la conexión. En este caso la modificación de los servicios puede ser realizada a un nivel específico de aplicación.

Los atributos del framework La Tempestad permiten a los proveedores de servicio convertirse efectivamente en operadores de redes, para alguna partición definida de la red física. Esto permite tomar ventaja de los conocimientos que poseen acerca de cómo los recursos de la red se van a utilizar, mediante la programación de su propia arquitectura de control (Van der Merwe, Cambridge Univ., Rooney, Leslie, \& Crosby, 1998).

De acuerdo a Kovačević (2009), ForCES de IETF tiene como objetivo estandarizar una arquitectura de red distribuida programable abierta. La estandarización incluye la especificación del protocolo para la comunicación entre el plano de control y reenvío en el router. Así mismo, ForCES permite la innovación rápida, tanto en el plano de control como en el plano de reenvío manteniendo la interoperabilidad (Yang, Dantu, Anderson, \& Gopal, 2004).

Según Sezer et al. (2013), Ethane es una arquitectura de gestión de seguridad en la cual se combina los switches basados en flujo simple con un controlador central y esto permite la administración de la admisión y enrutamiento de los flujos. Además el controlador central de Ethane crea una solución lógica a nivel de flujo para el control de acceso en redes empresariales, reduciendo así los switches en tablas de flujo que son pobladas por el controlador (Feamster, Rexford, \& Zegura, 2014).

El proyecto Ethane de Stanford permitió que sentaran las bases para la creación de OpenFlow, desarrollándose en un programa de Internet seguro cuyo objetivo era ofrecer una red programable para probar nuevos protocolos en las plataformas actuales de internet (Sharma, Staessens, Colle, Pickavet, \& Demeester, 2012). De acuerdo a OFN (Open Fundation Networking) describe que en la arquitectura de SDN, los planos de datos y control están desvinculados; el estado e inteligencia de la red están centralizados lógicamente, y la infraestructura de la red subyacente se abstrae de sus aplicaciones (ONF, 2012). OpenFlow consiste en dos entidades principales, primero el switch OpenFlow el cual implementa el plano de reenvío y segundo es el controlador el cual se implementa el en plano de control. De esa manera las dos entidades se comunican a través de un canal seguro que se ejecuta en un protocolo especial también llamado OpenFlow; debido a esto cada paquete que llega al switch que pasa a través de una serie de tablas de flujo o Flow Tables como se aprecia en la figura 2, la cual Goransson y Black (2014), la representan como estructuras de datos fundamentales en SDN que permiten evaluar los paquetes recibidos y tomar la decisión apropiada en base al contenido del paquete que llega al dispositivo SDN. Si la tabla de flujo encuentra una coincidencia se realiza una acción en el paquete en la cual el mismo puede ser llevado a otra tabla de flujo, o a un puerto de salida, o que se elimine por completo.

El controlador configura la operación del switch de OpenFlow mediante la utilización de sus tablas de flujo en donde una entrada comprende reglas y un conjunto de acciones e instrucciones. Dando así paso a una búsqueda que se realiza en cada trama oframe que llega según al parámetro de regla que utilice y en caso de coincidencia se llevan a cabo las acciones apropiadas y cada acción define qué operación se va a realizar en la trama entrante y una instrucción define de qué manera aplicar las acciones (e.g., aplique ahora, aplique después, etc.) (Gelberger, Yemini, \& Giladi, 


\section{Figura 2. \\ El esquema lógico del switch de OpenFlow basado en la arquitectura SDN.}

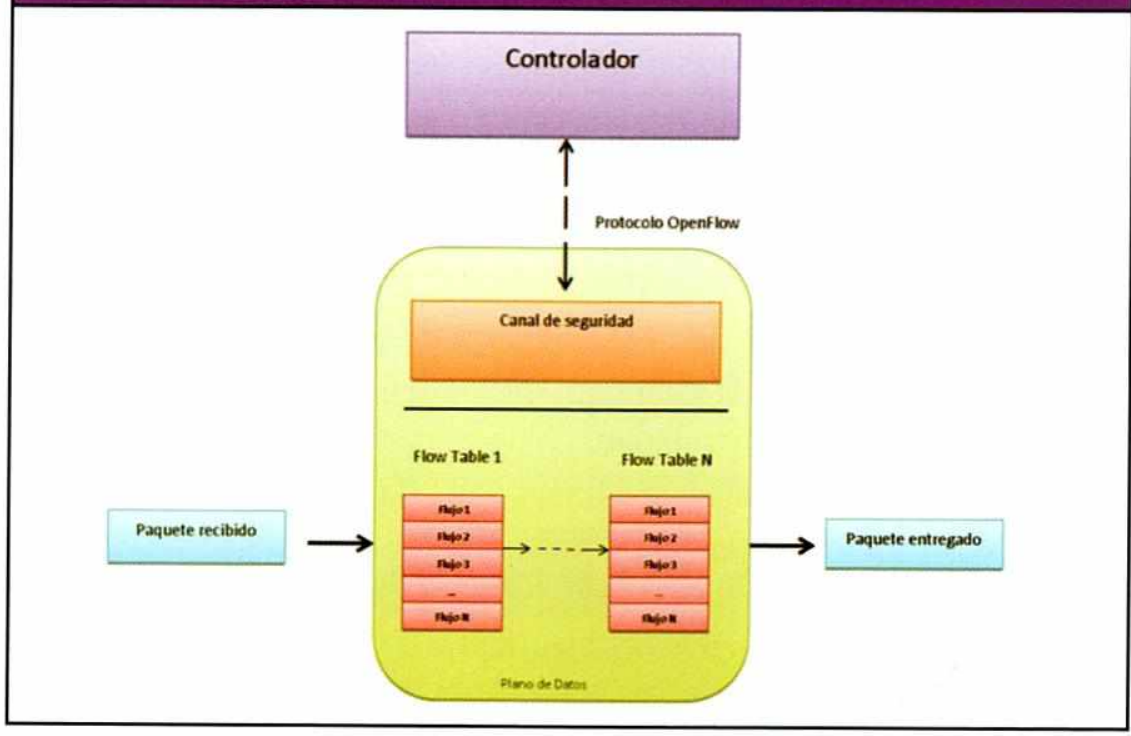

Fuente: Elaboración propia.

\section{Arquitectura de SDN}

De acuerdo a lo visto anteriormente, SDN es una arquitectura de red emergente donde el control está desasociado al reenvío y en la cual la red es programable directamente. La figura 3 muestra una vista lógica de la arquitectura de SDN en donde la inteligencia de la red (lógicamente) centralizada en los controladores es la que mantiene la vista global de la red y como resultado aparece como un único y lógico switch hacia las aplicaciones y motores de búsque- da (ONF, 2012). Con SDN, empresas y operadoras ganan independencia de los vendedores de software privados sobre las redes desde un punto de vista lógico, lo cual simplifica en gran medida la operación y el diseño de la red y también reduce los dispositivos de red en sí, puesto que ellos no necesitan comprender y procesar todas las normas de protocolos sino simplemente aceptar instrucciones provenientes de los controladores de SDN (Kumar, 2014; ONF, 2012).

\section{Figura 3.}

Esquema de la arquitectura de SDN. Adaptado de "Software-Defined Networking: The New Norm for Networks", por ONF, 2012.

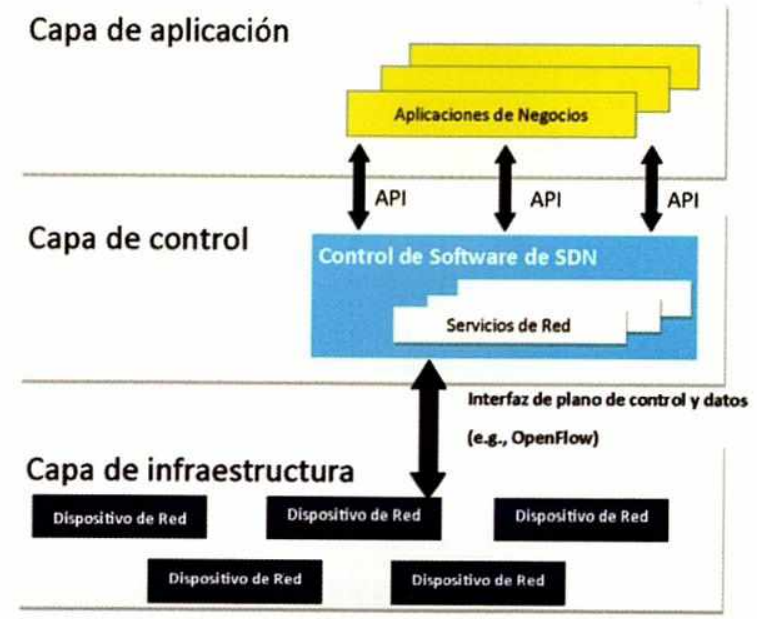




\section{Controladores}

Existen en el mercado un gran número de implementaciones de los controladores de SDN entre ellos se encuentra controladores basados en código abierto y comerciales (Goransson \& Black, 2014; McNickle, 2014). En la tabla 3, (como coinciden Casado, s.f; Shalimov, Zuikov, Zimarina, Pashkov, \& Smeliansky, 2013; Velrajan, 2012) se demuestra una lista de controladores de SDN con su lenguaje de programación, el cual se basa en Openflow debido a que trabajan como sistemas abiertos. Sin embargo existen controladores de SDN privados en donde se ve que las empresas comerciales tales como Cisco, VMware y NEC se encuentran a la expectativa de explotar SDN al éxito financiero el cual se espera que este sea capaz de proporcionar mejor funcionalidad a los sistemas heredados que provee la comunidad de código abierto para desarrollar gran parte de SDN en base al software de la capa de control (Goransson \& Black, 2014).

\section{Tabla \# 3}

\section{Controladores de SDN basados en OpenFlow}

\begin{tabular}{|c|c|}
\hline Controlador & Lenguaje de Programación \\
\hline POX & Python \\
\hline Iris & Java \\
\hline MUL & C \\
\hline NOX & C++ y Python \\
\hline Jaxon & Java \\
\hline Trema & C y Ruby \\
Beacon & Java \\
\hline Floodlight & Java \\
\hline Maestro & Java \\
\hline
\end{tabular}

Nota: Adaptado de "Controller Platforms", por M. Casado, s.f, McKeown Group.

Con respecto a la figura 4 se puede observar que SDN tiene una Interfaz de programación de aplicaciones o Application Programming Interface (API) que permite a los administradores centralizar el programa y manejar los recursos de la red a través de aplicaciones y servicios. Esto permite a los administradores de red programar todo en el plano de control a través de una API común, de tal manera que facilita el proceso y permite control más flexible sobre los flujos de tráfico y las políticas de la red utilizan para administrar (Ortiz Jr., 2013). En la figura 4 se muestra la dirección de la interfaz que vincula la capa de control con el nivel de la capa de infraestructura en la cual se define como el Southbound API. Así mismo se vincula la capa de aplicación con la capa de control se define como Northbound API (Sezer et al., 2013). 


\section{Figura 4.}

Ejemplo de la arquitectura de SDN donde Northbound y Southbound permiten la vinculación con los controladores. Adaptado de "An example of SDN architecture", por K, Kirkpatrick, 2013, Software-Defined Networking, p.17. Copyright 2013 por Communications of the ACM.

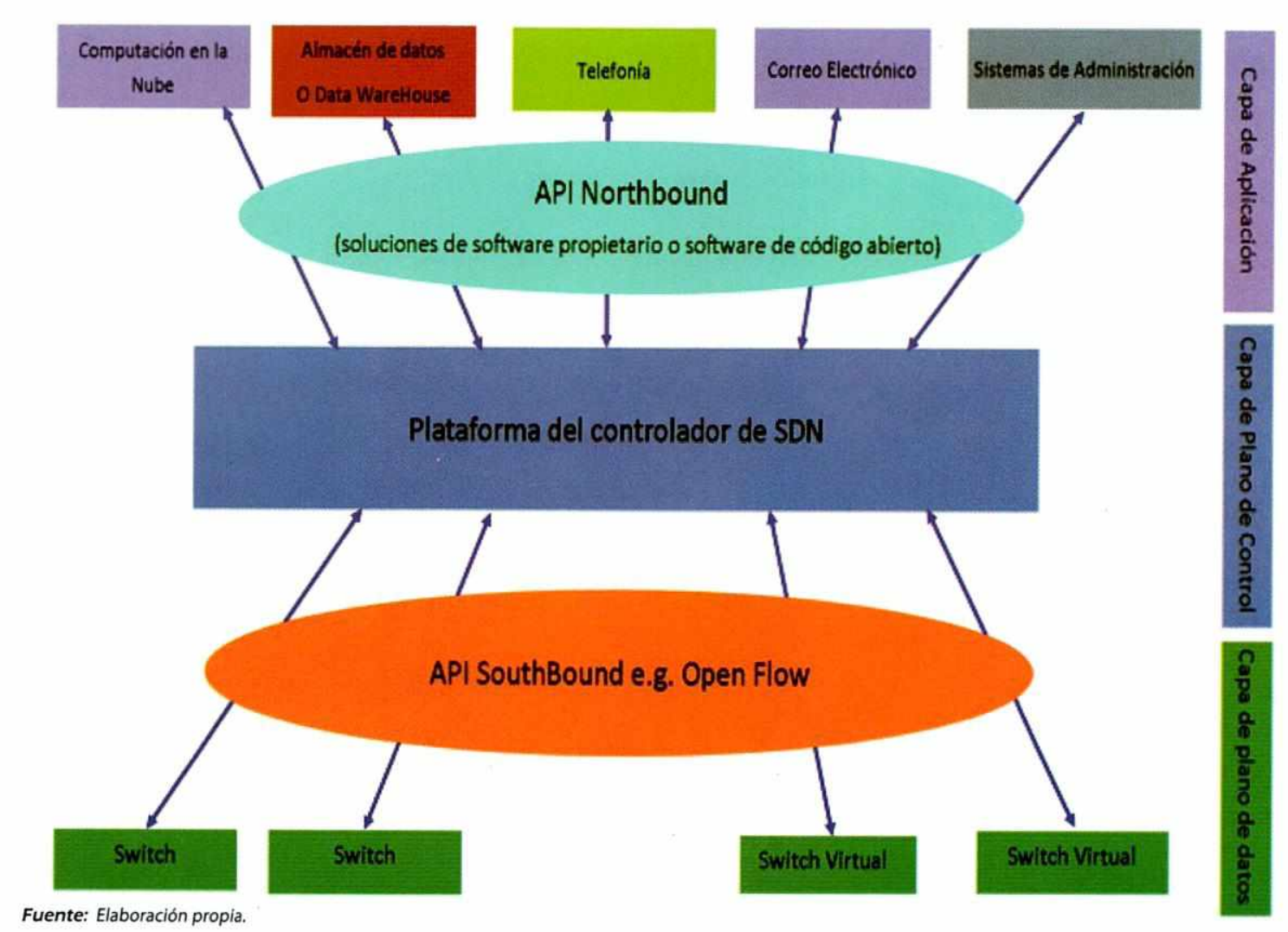

\section{API Northbound}

Se conecta con el controlador en donde permite que las aplicaciones y el sistema general de gestión programen la red y los servicios de petición, además este nivel de aplicación incluye a menudo la automatización y gestión de datos de aplicaciones globales, así como proporcionar funciones de red, tales como la computación de ruta de datos, enrutamiento y seguridad. En la actualidad, las normas estandarizadas no han sido ratificadas para el API Northbound con varias docenas de protocolos abiertos y de software propietario que están siendo desarrolladas utilizando diferentes APIs Northbound (Kirkpatrick, 2013). La falta de un API estándar se debe probablemente a la variada naturaleza de las aplicaciones que se encuentran encima del controlador
(Qin, Denker, Giannelli, Bellavista, \& Venkatasubramanian, 2014), como la gestión de sistemas de correo electrónico, servicios de computación en la nube, virtualización de los sistemas de red y otras funciones especializadas.

\section{API Southbound}

De acuerdo a Jarschel, Wamser, Hohn, Zinner, y Tran-Gia (2013), el API Southbound permite la externalización de la capa de control con la capa de infraestructura en una red lógicamente centralizada. SDN no indica expresamente a OpenFlow como un protocolo de uso frecuente pero según Nadeau y Gray (2013), provee un API Southbound estandarizado el cual se define como un conjunto de comandos abiertos para el reenvío de datos donde los comandos 
permiten a los routers descubrir la topología de la red. Además define el comportamiento de los switches físicos y virtuales, con base en los servicio de petición de solicitudes de aplicaciones enviados a través de las APIs Northbound. OpenFlow se usa comúnmente en la arquitectura de SDN, pero las organizaciones pueden optar por otros tipos de APIs Southbound para el control de los switches y dispositivos de acuerdo a los parámetros que ellos deseen (Kirkpatrick, 2013).

\section{Estrategias de implementación de SDN}

Como indica Raza et al. (2014), la implementación de estrategias de SDN reúne empresas o proveedores que ofrecen diferentes productos como se muestra en la tabla 4, donde se aprecia la estrategia con implementación de SDN como propietario o software propietario, el cual le da importancia a la capacidad de la programación. Sin embargo restringe el uso de código abierto en una estructura programable, debido a que utiliza componentes de pro- piedad privada. Se encuentran proveedores como Cisco y VMWare que venden sus propios controladores de SDN junto con las aplicaciones de software de alto nivel, como parte de su sistema de red programable.

Se muestra las estrategias de SDN que están basadas en el concepto de no propietario y código abierto, en donde el propósito principal de SDN es que el código abierto esté al alcance de todos sin componentes de propiedad privada, para que el uso y desarrollo independiente de SDN pueda crecer más (Raza et al, 2014).

De la misma manera Darbha et al. (2013) declaran que existen proveedores de dispositivo de red que están adoptando la arquitectura de SDN con software de código abierto, como OpenFlow y además existen empresas como HP, Big Switch y Juniper, que han adaptado la arquitectura SDN en colaboración con OpenFlow para la creación de dispositivos híbridos que sean compatibles en la red.

\section{Tabla \# 4}

\section{Lista de proveedores de dispositivos de red que implementan la estrategia SDN}

\begin{tabular}{|l|l|l|c|}
\hline Proveedor & Producto & $\begin{array}{l}\text { Estrategia } \\
\text { SDN } \\
\text { Propietario }\end{array}$ & $\begin{array}{c}\text { Estrategia SDN } \\
\text { (Código abierto y } \\
\text { No propietario }\end{array}$ \\
\hline Big Switch & $\begin{array}{l}\text { Big Switch Networks } \\
\text { Open SDN Suit }\end{array}$ & & $\checkmark$ \\
\hline Cisco & $\begin{array}{l}\text { One Platform Kit } \\
\text { (OnePK) }\end{array}$ & $\checkmark$ & \\
\hline Juniper & Open Contrail & $\checkmark$ & \\
\hline Hewlett-Packard & Open Flow enabled & & $\checkmark$ \\
\hline Linux Foundation & Open Daylight & & \\
\hline VMWare & NSX & $\checkmark$ & \\
\hline
\end{tabular}

Nota: Adaptado de "Propietary SDN Strategy," por M. H. Raza, S. C. Sivakumar, A. Nafarieh, \& B. Robertson, 2014. A Comparison of Software Defined Network (SDN) Implementation Strategies, p.1053-1054. Copyright 2014 por Procedia Computer Science. 


\section{Seguridad de SDN}

Según indica Douligeris y Serpanos (2007), las propiedades básicas de una red de comunicación segura son: confidencialidad, integridad, disponibilidad de la información, autenticación y no repudio. Hecha la observación anterior un modelo de negocio de telecomunicaciones que implemente $\mathrm{SDN}$, es vulnerable a las amenazas potenciales a causa del modelo de programación de SDN, en el cual el controlador se ve comprometido, debido a su modo centralizado lógico en donde puede ser objeto de mal uso, si existe algún ataque que permita el acceso o explotación no autorizada (Gupta, 2013; Metzler, 2012; ONF, 2013).

En SDN existen vulnerabilidades en la seguridad de la plataforma en donde Scott-Hayward et al. (2013) declaran que se encuentran al nivel de la capa del controlador con la capa de aplicación y al nivel de la capa del controlador con la capa de infraestructura. Por consiguiente muestra soluciones que proponen resolver la situación de vulnerabilidad, permitiendo así a los ingenieros programar filtros más granulares y capacidades de asignación de puertos, para emular la funcionalidad tradicional apertura o cierre del flujo en los switches SDN para el monitoreo de la seguridad.

\section{Casos de éxito}

Existen diferentes empresas que han adoptado SDN para el desarrollo de las redes y para dar mejor soporte a los usuarios; por ejemplo Intel ha tomado como alternativa virtualizar su red de centro de datos para poder aumentar la agilidad de sus aplicaciones en la misma (Intel Corporation, 2014) y al igual que Intel, varias compañías han tomado la misma decisión para mejorar su rendimiento, tales como Oracle, Dell, IBM, Google, Cisco, Facebook, AT\&T, entre otras se encuentran invirtiendo en esta tendencia (Berndtson, 2012, Burt, 2012; Myers, 2012). De acuerdo a Vance (2013) se han creado empresas que pretenden lanzar SDN como una gran solución en la arquitectura de redes y tienen como meta crear estándares de código abierto de SDN. $\mathrm{Al}$ parecer la tendencia actual del mercado va dirigida hacia esta tecnología emergente.

\section{Metodología}

Se utiliza en el siguiente trabajo de investigación, el enfoque cualitativo y se emplea, como técnica de recolección de datos, la entrevista donde se presenta como tipo de entrevista individual, cuya modalidad es no estructurada (Rodríguez Gómez, Gil Flores, \& García Jiménez, 1999). Se realiza la operación de trascripción de oral a escrita a las fuentes de información (López Estrada \& Jean-Pierre, 2011).

Con referencia a lo anterior, para la obtención de la información se realiza las entrevistas con dos representantes de los principales portadores del Ecuador, según consta en la Supertel, Corporación Nacional de Telecomunicaciones y Telconet. Además se realiza una entrevista al gerente de Hewlett-Packward especialista en Networking para conocer la opinión de la problemática estudiada.

Posteriormente, para el análisis de los datos cualitativos, se categoriza las preguntas estipuladas a los entrevistados y se realiza un cuadro de comparaciones en base a los resultados de forma resumida. (Hernández Sampieri, Fernández Collado, \& Baptista Lucio, 2010). Finalmente, mediante el procesamiento de los datos se determina el estado actual de los portadores ante el uso de SDN.

\section{Análisis y resultados}

Una vez aplicado el método de recolección de datos, en la tabla 5 se procede a categorizar las preguntas efectuadas a los entrevistados, para realizar la matriz de comparaciones de empresas de telecomunicaciones del Ecuador, la cual permite el análisis de los mismos y da paso a las conclusiones de la investigación. Donde se muestra si SDN pueda servir como alternativa de solución para los servicios portadores del Ecuador. 


\section{Tabla \# 5}

\section{Categorización de las preguntas efectuadas a los entrevistados}

\begin{tabular}{|c|c|}
\hline Categoría & Pregunta \\
\hline $\begin{array}{l}\text { Conocimiento de } \\
\text { SDN }\end{array}$ & 1.- ¿Según su punto de vista, qué considera que es SDN? \\
\hline Implementación & $\begin{array}{l}\text { 2.- ¿En su empresa se ha implementado las redes definidas por } \\
\text { software? }\end{array}$ \\
\hline Dispositivos & 3.- ¿Existe equipos de su empresa que trabajan con SDN? \\
\hline $\begin{array}{l}\text { Alternativa de } \\
\text { Solución (tiempo) }\end{array}$ & $\begin{array}{l}\text { 4.- ¿CNT como portador principal del Ecuador, cree usted que } \\
\text { SDN ofrece una alternativa de solución? }\end{array}$ \\
\hline Eficiencia & $\begin{array}{l}\text { 5.- ¿Considera usted a SDN como una solución eficiente tanto en } \\
\text { el ámbito económico como en los costos operacionales? }\end{array}$ \\
\hline Seguridad de SDN & $\begin{array}{l}\text { 6.- Dentro de la Ley Especial de Telecomunicaciones Reformada } \\
\text { resalta que El Plan de Desarrollo de las Telecomunicaciones tiene } \\
\text { por finalidad dotar al país de un sistema de telecomunicaciones } \\
\text { capaz de satisfacer las necesidades de desarrollo, para establecer } \\
\text { sistemas de comunicaciones eficientes, económicas y } \\
\text { seguras. ¿Considera usted que SDN brinda seguridad para que no } \\
\text { sea vulnerable el sistema? }\end{array}$ \\
\hline Estrategia de SDN & $\begin{array}{l}\text { 7.- ¿Qué estrategias de implementación de SDN utilizaría? } \\
\text { ¿Código abierto o Software propietario? }\end{array}$ \\
\hline $\begin{array}{l}\text { Comparación con } \\
\text { el modelo OSI }\end{array}$ & $\begin{array}{l}\text { 8.- ¿Cuál cree que es la comparación del modelo de la capa OSI } \\
\text { con el modelo de SDN? }\end{array}$ \\
\hline
\end{tabular}

Nota: La categorización permite una mayor comprensión de los datos.

Fuente: Elaboración propia

Para el análisis de los datos de la tabla 6, se agrupó las categorías de forma alfabética en contraste con los resultados de los entrevistados, donde las entrevistas se encuentran en el anexo 1 de este documento. Los resultados de las entrevistas fueron abiertas y se procedió a hacer un resumen de las mismas de acuerdo a su categoría. 
Tabla \# 6

\section{Cuadro de comparaciones de las categorias con las empresas de telecomunicaciones}

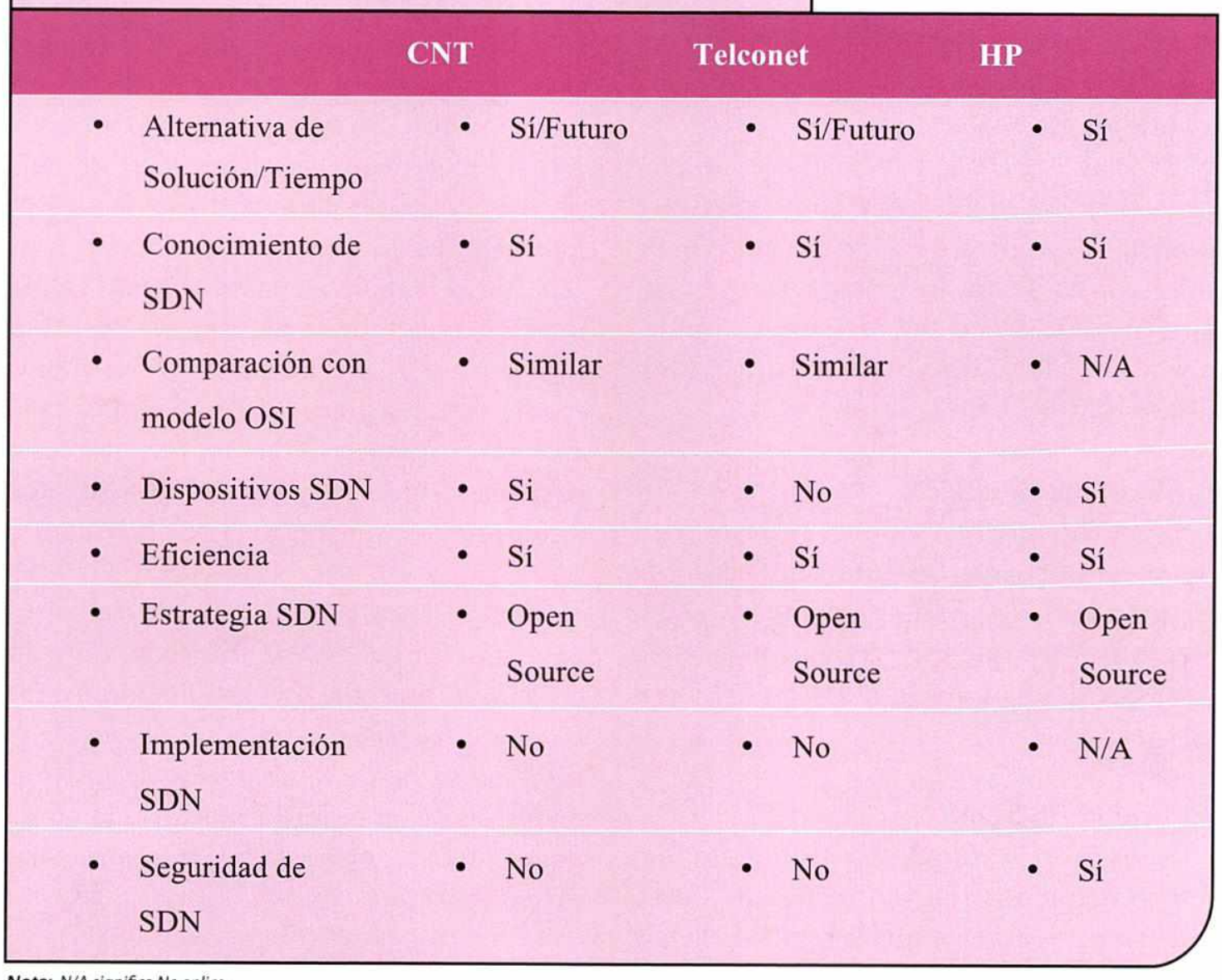

\section{Alternativa de solución}

Se les preguntó a los entrevistados si consideran a SDN como alternativa de solución para el Ecuador y todos coincidieron que sí. Las principales empresas de telecomunicaciones que ofrecen servicios portadores optan por el sí, pero no en el presente (Montero, 2014; Yépez, 2014). En cambio en HP Networking (Vivar, 2014) aceptan a SDN como alternativa de solución debido a que es un vendedor de dispositivos en base a SDN.

\section{Conocimiento de SDN}

Se les preguntó su opinión acerca de SDN a los entrevistados y cada uno presentó conocimiento amplio con respecto a la arquitectura, estrategias de implementación y seguridad de SDN (Vivar, 2014; Montero, 2014; Yépez, 2014).

\section{Comparación con modelo OSI}

CNT y Telconet consideran que la capa del modelo OSI es similar al del modelo de SDN (Montero, 2014; Yépez, 2014), mientras que HP Networking no aplica debido a que no se pudo hacer la pregunta al entrevistado.

\section{Dispositivos SDN}

CNT y Telconet no poseen dispositivos en base a SDN para la gestión de red en los servicios portadores (Montero, 2014; Yépez, 2014). En cambio HP Networking (Vivar, 2014), como vendedor de tecnología ofrece equipos que trabajen con SDN. 


\section{Eficiencia de SDN}

De acuerdo a los entrevistados, las redes definidas por software sí presentan una solución eficiente correspondiente a los costos operacionales en una red (Vivar, 2014; Montero, 2014; Yépez, 2014).

\section{Estrategia de SDN}

Existen varias estrategias de implementación de SDN, tales como software propietario y software Open Source o de código libre. Todos los entrevistados resaltan el uso de Open Source para la implementación de redes SDN (Vivar, 2014; Montero, 2014; Yépez, 2014).

\section{Implementación SDN}

Se puede observar que en la actualidad los portadores no han implementado la arquitectura de SDN (Montero, 2014; Yépez, 2014), y que HP Networking siendo vendedor de equipos de SDN no aplica en esa categoría.

\section{Seguridad de SDN}

Los servicios portadores, siendo grandes empresas que distribuyen tráfico de datos, tienen muy pendiente que la seguridad en la implementación de SDN es primordial. CNT y Telconet respondieron que no creen que SDN brinde seguridad al sistema (Montero, 2014; Yépez, 2014). Sin embargo HP Networking considera que si lo hace (Vivar, 2014).

\section{Conclusiones}

En este trabajo se presenta una arquitectura vanguardista llamada redes definidas por software, que permite mostrar una nueva forma de administrar las redes de telecomunicaciones. La creciente demanda por acceso a internet está causando un impacto global, de manera que este reciente manejo de las mismas no solo se extiende a las grandes operadoras que distribuyen internet; sino también a cualquier empresa o entidad que desee configurar, reforzar y mejorar las redes de tal forma que optimice la misma y promueva una mayor oferta de acceso a internet.

En Ecuador la demanda de acceso a internet también se ha incrementado; se puede entender que los servicios portadores del país, los cuales son los principales entes de transmisión de señales y datos, llevan una relación entre el número de usuarios y el actual crecimiento de la cantidad de enlaces para satisfacer el incremento de acceso a internet. Tales portadores deberán ir de acuerdo al Plan de Desarrollo de las Telecomunicaciones, donde se promueve el uso de sistemas de comunicaciones eficientes, económicas y seguras. Sin embargo, se coincide con Montero (2014) en que los costos para fortalecer y mejorar las redes son altos y comprenden mayor nivel de dificultad.

Para el natural desarrollo del trabajo se debe conocer que SDN fue creado como una tendencia que fue evolucionando con el pasar de los años, donde sus inicios comprendieron desde laboratorios de universidades, hasta grandes organizaciones mundiales que se unen a favor de optimizar el nuevo concepto.

Por consiguiente, se muestra la arquitectura de SDN en donde la red se divide en capas; tales están desasociadas entre ellas, pero cada una cumple con una función importante en donde la interacción entre ellas es a través de las interfaces APIs Northbound y Southbound. Estas trabajan con controladores, que son la base fundamental de la red, donde se apoyan en políticas de control y gran variedad de plataformas de lenguaje que lo hace accesible a cualquiera que desee utilizarlo para la optimización de la red.

Los presentes controladores pueden ser de grandes empresas comerciales o no comerciales, como por ejemplo los controladores que HP Networking distribuye en Ecuador.

En SDN la interfaz Northbound ofrece la interacción de la capa de control con la 
capa de aplicación, pero no existe una estandarización con respecto al uso del mismo. En cambio la interfaz Southbound que trabaja en la interacción entre la capa de control, con la capa de infraestructura, presenta una estandarización por medio de OpenFlow pero no es la única opción.

Para los servicios portadores existe una gran variedad de vendedores de dispositivos SDN, en donde pueden utilizar las estrategias propietario o estrategias no propietario y de código abierto.

El beneficio de utilizar una estrategia SDN en base al concepto propietario, implica mayor costo, mejor soporte y seguridad, pero limita la libre programación de la red. En cambio el beneficio de utilizar una estrategia no propietario y de código abierto, permite la libre implementación de la programación de la red, disminuye el costo de equipos, pero la seguridad se ve comprometida.

Cualquiera que sea la implementación que se utilice, los servicios portadores deben tener en cuenta que para mejorar la red, se debe promover la seguridad, la eficiencia de comunicación y la economía. Además si se acepta SDN como una solución para reforzar o mejorar la red, se debe considerar el mismo como una nueva tendencia que va adquiriendo nuevos adeptos; tales como Google, Cisco, entre otros. Por lo tanto va acaparando más mercado y mayor soporte; a pesar de su crecimiento como nueva tecnología puede presentar fallas a los nuevos problemas de seguridad y a la interacción con todos los protocolos.

En el caso ecuatoriano, SDN aun no ha sido implementado en dos de los principales servicios portadores, pero puede proveer una alternativa de solución en el futuro. Además se puede indicar la preferencia de los mismos al considerar la estrategia de implementar SDN en base a Open Source.

Adicionalmente se puede concluir que los portadores consideran a SDN como solu- ción eficiente que disminuye los costos operacionales de la ejecución del sistema.

Sin embargo, se debe contemplar la implementación en base a la estrategia de SDN propietario, debido a que ofrece garantía de estabilidad para los clientes de las portadoras y brinda soporte a los grandes proveedores de dispositivos.

En el presente trabajo, aunque se realizó las entrevistas a dos de los principales portadores del Ecuador y a un distribuidor de equipos de red, para el análisis de la situación actual, se presentó como limitante el no haber tomado como referencia a las demás portadoras. En ese sentido se recomienda hacer el estudio del estado, administración e implementación actual de los servicios portadores; de tal manera que permita analizar la posible inclusión de SDN en sus redes respectivas. De igual forma se recomienda buscar el seguimiento de la estandarización de SDN para futuros proyectos de telecomunicaciones, que permita crecer a esta en el Ecuador, no solo en el avance de las investigaciones, sino también en ser fuente de soluciones de esta nueva tecnología, para poder venderla de forma nacional e internacional. Además se sugiere realizar investigaciones que permitan comparar el uso de software libre, con el comercial, debido al gran recibimiento que se presenta en las comunidades de software libre. Finalmente se recomienda como futura investigación buscar nuevos campos que SDN está trabajando y adaptarlo al entorno, no solo de las telecomunicaciones, sino también a los servicios relacionados a la nube o los data center que están surgiendo en Ecuador.

\section{Referencias Bibliográficas}

Barkai, S. (10 de octubre de 2013). Do Mobile Carriers Need SDN? Recuperado el 11 de octubre de 2014, de SDN Central: https:/www.sdncentral.com/education/mobile-carriers-need-sd$\mathrm{n} / 2013 / 10 /$ 
Berndtson, C. (30 de abril de 2012). 25 Software-Defined Networking Player To Know. CRN. Obtenido de http://www.crn.com/slide-shows/networkin g/232900998/25 - s of t ware-defined-networking-players-to- $k$ now.htm/pgno/0/20

Burrows, P. (9 de junio de 2014). Cisco Challenged as Facebook Favors Software to Move Data: Tech. Recuperado el 11 de octubre de 2014, de Bloomberg: h t t p : / / w w w b loo m ber g. com/news/2014-06-10/cisco-challenged-as-facebook-favors-software-to-move-data-tech.html

Burt, J. (27 de abril de 2012). Enterprise Networking: How Cisco, HP, Juniper; Others Are Tackling SDN, OpenFlow. Recuperado el 11 de octubre de 2014, de Eweek: http://www.eweek.com/c/a/ Enterprise-Networking/How-Cisco-HP-Juniper-Others-Are-Tackling-SDN-OpenFlow-183460/

Casado, M. (s.f). Controller Platforms. Recuperado el 10 de octubre de 2014, de McKeown Group: http://yuba.stanford.edu/ casado/of-sw.html

Cisco Systems Inc. (5 de febrero de 2014). Cisco Visual Networking Index: Global Mobile Data Traffic Forecast Update. Recuperado el 16 de septiembre de 2014, de Cisco System Inc: http://www.cisco.com/c/en/us/solutions/collateral/service-provider/visual-networkingindex-vni/white_paper_c11-520862. html

CITRIX. (s.f de mayo de 2014). SDN 101: An Introduction. Recuperado el 11 de octubre de 2014, de CITRIX: http:// www.citrix.com/content/dam/citrix/en_us/documents/oth/sdn-101-anintroduction-to-software-defined-networking.pdf
Crabbe, E. (octubre de 2011). About Tech Field Day. Obtenido de sitio web Tech Field Day: http://static.techfieldday.com/wp-content/uploads/ 2011/10/TheLongRoadtoSDN.pdf

Darbha, S., Shevenell, M., \& Normandin, J. (2013). Impact of Software-Defined Networking on . CA Technologies, 33-43.

Davy, M. (julio de 2011). A case for expanding OpenFlow/SDN! Recuperado el 11 de octubre de 2014, de OpenFlow: http://archive.openflow.org/w $\mathrm{p} / \mathrm{wp}$-content/uploads/2011/07/GENI-Workshop-Whitepaper.pdf

Douligeris, C., \& Serpanos, D. N. (2007). Network security: current status and future directions. John Wiley \& Sons.

Farrel, A., Vasseur, J.-P., \& Ash, J. (agosto de 2006). About us: The Internet Engineering Task Force. Recuperado el 23 de septiembre de 2014, de sitio web de The Internet Engineering Task Force: http://tools.ietf.org/html/rfc4655

Feamster, N., Rexford, J., \& Zegura, E. (2014). The road to SDN: an intellectual history of programmable networks. ACM SIGCOMM Computer Communication Review, 44(2), 87-98.

Feng, T., Bi, J., \& Wang, K. (2014). Joint allocation and scheduling of network resource for multiple control applications in SDN. IEEE, 1-7.

Gelberger, A., Yemini, N., \& Giladi, R. (2013). Performance Analysis of Software-Defined Networking (SDN). Analysis \& Simulation of Computer and Telecommunication Systems (MASCOTS), 2013 IEEE 21st International Symposium on Modelling (págs. 389-393). Beer-Sheva: IEEE. 
Goransson, P., \& Black, C. (2014). Software Defined Networks: A Comprehensive Approach. Waltham: Elsevier Science.

Gupta, L. (13 de diciembre de 2013). SDN: Development, Adoption and Research Trends. Recuperado el 10 de octubre de 2014, de Washington University: http://www.cse.wustl.edu/ jain/ cse570-13/ftp/sdn.pdf

Hernández Sampieri, R., Fernández Collado, C., \& Baptista Lucio, P. (2010). Recolección y análisis de los datos cualitativos. En Metodología de la investigación (págs. 406-487). México D.F: McGraw-Hill.

INEC. (12 de septiembre de 2014). Tecnologías de la Información y Comunicaciones (TIC'S) 2013. Recuperado el 10 de octubre de 2014, de Instituto Nacional de Estadística y Censos: http://www.observatoriotic.mintel.gob. ec/images/varios/Noticias/Reportes/Resultados_principales_12_09_2014.pdf

Intel Corporation. (abril de 2014). Adopting Software-Defined Networking in the Enterprise. Obtenido de IT@ Intel White Paper: http://www.intel.com/content/dam/www/public/us/en/documents/best-practices/adopting-software -defined-networking-in-the-enterprisepaper.pdf

Internet Live Stats. (1 de julio de 2014). Usuarios de internet: Internet Live Stats. Recuperado el 15 de septiembre de 2014, de Sitio Web de Internet Live Stats: http://www.internetlivestats.com /internet-users/

Internet World Stats. (2013 de diciembre de 2013). Internet Growth Statistics. Obtenido de Internet World Stats: http:// www.internetworldstats.com/stats. htm
Jarschel, M., Wamser, F., Hohn, T., Zinner, T., \& Tran-Gia, P. (2013). Sdn-based application-aware networking on the example of youtube video streaming. 2013 Second European Workshop on Software Defined Networks (EWSDN) (págs. 87-92). Würzburg: IEEE.

Kirkpatrick, K. (2013). Software-Defined Networking. Communications of the ACM, 16-19.

Kobayashi, M., Seetharaman, S., Parulkar, G., Appenzeller, G., Little, J., Reijendam, J. V., . . . McKeown, N. (2014). Maturing of OpenFlow and Softwaredefined Networking through deployments. Computer Networks, 151-175.

Kovačević, I. (2009). FoRCES protocol as a solution for interaction of control and forwarding planes in distributed routers. 17th Telecommunications fortum TELFOR 2009 (págs. 529-532). Belgra- de: Telfor Journal.

Kumar, K. (26 de febrero de 2014). Virtually Networked: The State of SDN. Recuperado el 10 de octubre de 2014, de The Viodi View: http://viodi.com/ 2014/ 02/26/virtually-networked-the-state-of-sdn/

López Estrada, R. E., \& Jean-Pierre, D. (junio de 2011). La entrevista cualitativa como técnica para la investigación en Trabajo Social. Obtenido de Margen 61: http://www.margen.org/suscri/margen61/lopez.pdf

McNickle, M. (18 de septiembre de 2014). Five commercial SDN controllers to know about. Recuperado el 10 de octubre de 2014, de Tech Target: http://s e a r c h s d n.tech t a r get.com/news/2240230895/Five-commercial-SDN-controllers-to-knowabout 
Metzler, J. (9 de septiembre de 2012). Research: Understanding Software-Defined Networks. Recuperado el 10 de octubre de 2014, de Information Week Reports: http://reports.informationweek.com/abstract/6/9044/Data-Center/research-understandingsoftware-defined-networks.html

Montero, C. (31 de octubre de 2014). Redes Definidas por Software. (G. N. Cuenca Pérez, Entrevistador)

Montero, C. (31 de octubre de 2014). Redes definidas por Software en Ecuador. (G. Cuenca, Entrevistador) Guayaquil, Guayas.

Myers, A. (17 de mayo de 2012). OPEN NETWORKING SUMMIT TO EXPLORE SOFTWARE-DEFINED NETWORKING. Recuperado el 11 de octubre de 2014, de Stanford University: h t t p://engineering.stan ford. edu/news/open-networking-summit-explore-software-defined-networking

Nadeau, T. D., \& Gray, K. (2013). SDN: Software Defined Networks. Sebastopol: O'Reilly Media, Inc.

Newman, P., Edwards, W. L., Hinden, R. M., Hoffman, E., Liaw, F. -C., Lyon, T., \& Minshall, G. (agosto de 1996). Ipsilon's General Switch Management Protocol Specification. Obtenido de Internet Engineering Task Force: http://tools.ietf.org/html/rfc1987

ONF. (13 de abril de 2012). Software-Defined Networking: The New Norm for Networks. Recuperado el 20 de septiembre de 2014, de Open Networking Foundation: https://www.opennetworking.org/images/stories/downloads/sdn-resources/white-papers/wp-s dn-newnorm.pdf
ONF. (8 de octubre de 2013). SDN Security Considerations. Recuperado el 10 de octubre de 2014, de Open Networking Foundation: https:/www.opennetworking.org/images/stories/downloads/sdn-resources/solution-briefs/sbsecurity-data-center.pdf

Ortiz Jr., S. (2013). Software-Defined Networking: On the Verge of a Breakthrough? IEEE Computer Society, 10-12.

Parol, P., \& Pawlowski, M. (2013). Towards networks of the future: SDN paradigm introduction to PON networking for business applications. Proceedings of the 2013 Federated Conference on Computer Science and Information System (págs. 829-836). Kraków: IEEE.

Qin, Z., Denker, G., Giannelli, C., Bellavista, P., \& Venkatasubramanian, N. (2014). A Software Defined Networking Architecture for the Internet-of-Things. 2014 IEEE Network Operations and Management Symposium (NOMS), 1-9.

Raza, M. H., Sivakumar, S. C., Nafarieh, A., \& Robertson, B. (2014). A Comparison of Software Defined Network (SDN) Implementation Strategies. Procedia Computer Science, 1050-1055.

Rodríguez Gómez, G., Gil Flores, J., \& García Jiménez, E. (1999). La Entrevista. En Metodología de la investigación educativa (págs. 167-184). Málaga: ALJIBE.

Scott-Hayward, S., O'Callaghan, G., \& Sezer, S. (2013). SDN security: A survey. 2013 IEEE SDN for Future Networks and Services (SDN4FNS) (págs. 1-7). Belfast: IEEE. 
Sezer, S., Scott-Hayward, S., Fraser, B., Lake, D., Viljoen, N., Finnegan, J., . . . Miller, M. (2013). Are We Ready for SDN? Implementacion Challenges for Software-Defined Networks. IEEE Communications Magazine, 36-43.

Shackleford, D. (28 de junio de 2013). Tech Target. Recuperado el 10 de octubre de 2014, de Estrategias de seguridad de SDN para prevenir ataques a la red: http://searchdatacenter.techtarget.com/es/reporte/Estrategias-de-seguridad-deSDN-para-prevenir-ataques-a-la-red

Shalimov, A., Zuikov, D., Zimarina, D., Pashkov, V., \& Smeliansky, R. (2013). Advanced study of SDN/OpenFlow controllers. Proceedings of the 9th Central \& Eastern European Software Engineering Conference in Russia (págs. 1-6). Moscow: ACM.

Sharma, S., Staessens, D., Colle, D., Pickavet, M., \& Demeester, P. (2012). OpenFlow: Meeting carrier-grade recovery requirements. Computer Communications, 656-665.

Shin, M.-K., Nam, K.-H., \& Kim, H.-J. (2012). Software-defined networking (SDN): A reference architecture and open APIs. 2012 International Conference on ICT Convergence (ICTC) (págs. 360-361). Daejon: IEEE.

SUPERTEL. (2010). Análisis de los aspectos que influyen en los costos del servicio de Internet en el Ecuador. Revista institucional de la Superintendencia de Telecomunicaciones, 11-13.

Tiwari, V. (2013). SDN and OpenFlow for beginners with hands on labs. M.M.D.D. Multimedia LLC.

Tomkins, R. (febrero de 2014). How software-defined networking will boost carrier Ethernet services. Recuperado el 11 de octubre de 2014, de Tech Target: http://searchsdn techtarget.com/opinion/Howsof tware-defined-networkingwill-boost-carrier- Ethernet-services
Van der Merwe, J., Cambridge Univ., U., Rooney, S., Leslie, I., \& Crosby, S. (Mayo de 1998). The Tempest-a practical framework for network programmability. IEEE Network, 20-28.

Vance, J. (25 de septiembre de 2013). Ranking the Top 10 Software Defined Networking Startups. CIO. Obtenido de http://www.cio.com/article/2383078/mergers-acquisitions/ranking-the-top-10-software-defined-networking-startups.html

Velrajan, S. (12 de diciembre de 2012). List of OpenFlow Controllers for SDN. Recuperado el 10 de octubre de 2014, de The Tech: h t t p : // w w w. the tech.in/2012/12/list-of-openflow-controllers-for-sdn.html

Vivar, J. (31 de octubre de 2014). Entrevista de G. Cuenca. Redes definidas por Software en Ecuador, Guayaquil, Guayas.

Yang, L. L., Dantu, R., Anderson, T. A., \& Gopal, R. (abril de 2004). Forwarding and Control Element Separation (ForCES) Framework. Obtenido de sitio Internet Engineering Task Force: http:// tools.ietf.org/ html/rfc3746

\section{Gabriela Cuenca Pérez}

Ing. en Telecomunicaciones de la Universidad Espiritu Santo - Ecuador

E-mail:gcuenca@uees.edu.ec

\section{Mónica Flores Marin}

Ing. en Electricidad, Especialización Electrónica y Automatización Industrial

Magister en Administración de Empresas

Docente tiempo completo de la Universidad Espiritu Santo - Ecuador

E-mail: mfloresm@gmail.com 


\section{Anexos}

\section{Anexo \#1: \\ Entrevistas}

La entrevista se realizó a un especialista en el área de RED IP/MPLS, el Ing. Eddy Yépez, quien actualmente es Analista de diseño de red de plataformas IP-MPLS en CNT.

1.- ¿Según su punto de vista, qué considera que es SDN?

SDN brinda la oportunidad de virtualizar una red, además que se puede obtener un mayor control de tráfico, mayor control eficiente y se puede crear instancias u objetos de lo que podría ser parte de la red.

2.- ¿En su empresa se ha implementado las redes definidas por software?

No se ha implementado las redes definidas por Software.

3.- ¿Existe equipos de su empresa que trabajan con SDN?

Nosotros como MPLS no, pero Nexus de Cisco sí, existe en el departamento de TI y se que van a poner en los nuevos data center que van a construir.

4.- ¿CNT como portador principal del Ecuador, cree usted que SDN ofrece una alternativa de solución?

Depende mucho de lo que estemos entregando a nuestros clientes, recientemente estamos implementando una red IP MPLS bastante grande y nuestros servicios son basados en eso, para implementar SDN, creo que hasta cierto punto debemos recuperar la inversión de la implementación MPLS para poder desarrollar una red SDN, pero eso es a nivel nacional. Tenemos otras áreas como ISP, donde tiene distribución de red y conjunto de servidores, creo que un futuro como proveedores de servicio si lo hemos de implementar.
5.- ¿Considera usted a SDN como una solución eficiente tanto en el ámbito económico como en los costos operacionales?

$\mathrm{Si}$, de hecho en la parte de la administración de una red bastante grande, yo creo que facilita y simplifica muchas cosas.

6.- Dentro de la Ley Especial de Telecomunicaciones Reformada resalta que El Plan de Desarrollo de las Telecomunicaciones tiene por finalidad dotar al país de un sistema de telecomunicaciones capaz de satisfacer las necesidades de desarrollo, para establecer sistemas de comunicaciones eficientes, económicas y seguras. ¿Considera usted que SDN brinda seguridad para que no sea vulnerable el sistema?

No estoy tan al tanto de SDN con respecto a la seguridad pero ya que actualmente existe una tendencia bastante fuerte a que los datos sean seguros, lo más probable sea que si.

7.- ¿Qué estrategias de implementación de SDN utilizaría? ¿Código abierto o Software propietario?

Yo personalmente soy partidario del open source, una porque se va a presentar menos costos de implementación y al ser abierto cualquier persona pueda mejorarlo y que mejor que tener 10 millones de personas, un ejemplo exagerado, desarrollando ese sistema a que simplemente se tenga a 1000 personas de un círculo cerrado desarrollando eso, el cual se limita bastante lo que es la expansión del desarrollo de ese tipo de tecnología, sin embargo muchas veces nuestros clientes nos van a decir: "¿sabes qué? a mí no me interesa el open source, si no que yo quiero algo que sea seguro y que sea certificado $100 \%$ de que sea muy bueno" entonces bajo esas exigencias quizá nos vayan a pedir ese tipo de cosas y que nosotros tengamos que obtener software licenciado, hardware licenciado con su costo referente. 
8.- ¿Cuál cree que es la comparación del modelo de la capa OSI con el modelo de SDN?

Para lo que es SDN, creo que desaparece un poco esa separación, pero tiene un tratamiento casi similar al modelo OSI. Pero eso sería una de las principales deficiencias, no le damos a los datos, a lo que son las aplicaciones un tratamiento con un tipo de capacidad a lo que es aplicación y otro a lo que es control, de pronto para lo que es control, para lo que son conmutación rápida de paquetes si necesitamos un hardware que soporte esa conmutación, pero quizá por mejorar ese hardware para esa conmutación rápida de paquetes no le damos una mayor capacidad para soporte de aplicaciones. Pero eso fue hace tiempo atrás, ahora el hardware está creciendo y mejorando constantemente, incluso con tecnologías de procesadores o de mayor cantidad de núcleos, da a pensar que ahora el hardware ya no está tan limitante como antes.

A continuación se realiza la entrevista al Ing. Carlos Montero, Gerente Nacional de Networking en Telconet S.A.

1.- ¿Según su punto de vista, qué considera que es SDN?

Actualmente en el esquema de conmutación de paquetes que es lo que prácticamente todas las redes portadoras utilizan, es cada dispositivo que esté en toda la red (si hablamos de una red de 50 dispositivos) pues los 50 dispositivos normalmente tienen una inteligencia propia lo que se conoce como plano de control, entonces todos los planos de control normalmente se programan de forma individual y corren en el equipo. Digamos a una red que está hablando BGP o OSPF entonces indica que cada router está configurado para hablar ese protocolo particularmente, cada router tiene una visión completa de la red y toma de decisiones de conmutación de paquetes en función de lo que su visión de la red le dice donde falló un enlace, a donde tengo que enviar ese paquete, que pasa cuando se cae el enlace. SDN es un paradigma completamente diferente donde todas esas funciones del plano de control sean centralizadas en un controlador, entonces lo que permite es que un equipo grande tenga una visión completa de la red y ese equipo a modo de cerebro va a estar ordenando a todos los demás equipos que van a hacer en funciones de forwarding de conmutación de paquetes, de acuerdo a lo que el cerebro central lo ha programado. Ya no hay una decisión local del router, sino una comunicación con el controlador para que este controlador pueda actualizar sus tablas de rutas, sus tablas de forwarding y decir bajo estas condiciones dirigir al router. Ya no hay decisión de cada uno de los equipos, ya no existe red de 50 equipos, ya no hay 50 planos de control, sino que existe uno solo que está unificado.

2.- ¿En su empresa se ha implementado las redes definidas por software?

En nuestra empresa no se ha implementado, pero estamos empezando a adquirir equipamiento para hacer pruebas de conceptos. Estamos empapando de la tecnología en cuanto a casos de éxito y marco teórico en general, pero no es algo que hemos implementado todavía. Casualmente Telconet tiene dos data center y por ahí queremos iniciar pruebas de concepto.

\section{3.- ¿Qué equipos de su empresa trabajan con SDN?}

Estamos pensando en hacer pruebas de concepto para los primeros meses del próximo año y lo haremos con plataformas abiertas.

\section{4.- ¿'Telconet como portador del Ecua-} dor, cree usted que SDN ofrece una alternativa de solución?

Claro que sí, pero el tema es que los portadores tenemos una infraestructura normalmente grande ya instalada y SDN es una 
tecnología bastante nueva. Por así decirlo, entonces dada la criticidad de los servicios, dado el marco regulatorio sobre los niveles de servicio que debemos ofrecer los portadores, la adopción de SDN pienso que ha de ser bastante lenta. Principalmente por todo el cambio que conlleva, por todo el potencial impacto que puede tener sobre los usuarios, de tal forma que si nos ofrece una herramienta de gestión muy simplificada en cuanto a que básicamente interactuamos con el controlador, pero también nos demanda a adquirir nuevas destrezas, nuevos compromisos de soporte con nuestros vendedores con quienes nos proveen equipos. Es definitivamente viable, creo que para allá vamos a ir pero va a hacer una opción (al menos en el tema de portadores) bastante lenta. Con algunas personas que he conversado de distintos vendors grandes (no solo aquí en el país sino fuera del país también) coincidimos que al menos al día de hoy ningún portador tiene en producción una red SDN. Quienes han invertido en SDN son los gigantes de contenido como Google, Facebook que tienen recursos, decenas de personas dedicadas a investigación y desarrollo donde elaboran su propio software, red SDN donde se manda a producción. Es un escenario completamente diferente, también la envergadura de un gigante como Google con la envergadura de un portador ecuatoriano o del exterior. No hay ningún caso de éxito documentado de portador que esté con una red SDN en producción, hacia sus usuarios finales para sus servicios generales.

\section{5.- ¿Considera usted a SDN como una} solución eficiente tanto en el ámbito económico como en los costos operacionales?

En estos momentos, creo yo que me faltan y en general nos faltan elementos de juicio como para decir un sí rotundo. Pensaría por las ventajas que ofrece que en efecto a mediano y largo plazo va a ser eficiente. Hay algunos costos de capital bastante grande en los operadores, el hardware tradicional de hoy no soporta SDN, no entiende lo que pueda hablar con un controlador. Básicamente hay costo de capital en cuanto a nueva infraestructura, costo de operación en cuanto a entrenamiento humano que hay que hacer (porque es completamente diferente a lo que hemos hecho). En general, pienso que en mediano y largo plazo los costos de operación de SDN deberán ser menores, pero al inicio hay una inversión grande que hacer. En cuanto a portadores vamos a ir adoptando eso despacio, pero no deseo ser el primero en adoptar SDN por los riesgos que conlleva. Nuestra principal meta (al menos en Telconet) es la estabilidad de los servicios justamente por esa cláusula de telecomunicaciones y también por nuestra propia visión del negocio.

6.- Dentro de la Ley Especial de Telecomunicaciones Reformada resalta que El Plan de Desarrollo de las Telecomunicaciones tiene por finalidad dotar al país de un sistema de telecomunicaciones capaz de satisfacer las necesidades de desarrollo, para establecer sistemas de comunicaciones eficientes, económicas y seguras. ¿Considera usted que SDN brinda seguridad para que no sea vulnerable el sistema?

El tema con SDN será tan seguro como la gente que lo haya podido programar, bajo ese esquema, absolutamente, todo va a tener vulnerabilidad, el tema es proteger a los controladores, hacer redundantes de tal forma que no exista un colapso para la red si es que algo llegara a suceder. En ese aspecto, SDN puede ser más riesgoso de lo que hacemos el día de hoy, dado que si comprometo el controlador, adiós a toda la red. La idea es que una vez que hayan sido descargadas las tablas, si es que algo pasa con los controladores, no haya actualizaciones en cuanto a nuevos caminos a tomar. Pero si algo pasa al controlador, que mantengan sus tablas programadas de tal forma que el tráfico continue. 
7.- ¿Qué estrategias de implementación de SDN utilizaría? ¿Open Source o Software propietario?

Las primeras pruebas van a ser en base a Open Source, todo será Open Source al inicio. Vamos a ver cómo nos va con ciertos equipos que trabajan con OpenFlow, mismos que pueden comunicarse con un controlador OpenFlow; es la forma que hemos pensado simplemente porque es un tema de concepto.

Además se realiza una entrevista al Ing. Johnny Vivar, especialista en el área de ventas de HP Networking en Ecuador, que da un punto de vista diferente como vendedor de equipos HP, que trabajan con la arquitectura SDN, los cuales utilizan el protocolo OpenFlow, entre el dispositivo y el controlador.

1.- ¿Desde cuándo HP ofrece soluciones en base a SDN en Ecuador?

SDN no es una tendencia en Ecuador sino que es una tendencia a nivel mundial, HP viene invirtiendo desde hace cuatro años. Cuando una tecnología se hace pública se viene trabajando mucho tiempo antes en los laboratorios de HP, para que hoy en dia pueda ser ofertado al usuario final.

2.- ¿Qué tipos de dispositivos su empresa ofrece con SDN?

El protocolo que debe conversar el equipo de red con el controlador se llama OpenFlow, hoy en día, existen muchos switches, desde accesos muy sencillos hasta switches de chasis muy avanzados que vienen con soporte de OpenFlow, que son diseñados pa- ra clientes portadores. Entonces tenemos un abanico completo de dispositivos que sopor$\tan \mathrm{SDN}$, además tenemos routers que soportan OpenFlow.

3.- ¿A qué empresa, HP brinda sus servicios SDN?

Existen varias empresas en el ámbito comercial que están interesados en SDN; por el lado educacional, la Escuela Superior Politécnica del Litoral están por comprar equipamiento y controlador SDN para que sus estudiantes puedan desarrollar aplicaciones sobre SDN.

4.- ¿Qué estrategias de implementación utilizan los equipos de SDN? ¿Código abierto o Software propietario?

HP siempre promueve los estándares de la industria; en ese sentido HP utiliza OpenFlow que es el protocolo estándar, no es nada propietario y no solo lo utiliza HP, sino también el resto de fabricantes interesados en utilizar un protocolo abierto.

5.- ¿Los dispositivos que trabajan con SDN están preparados para dar seguridad a la empresa?

En realidad es un dispositivo más, todo va a depender de cómo sea configurado, de cómo han de ser las políticas de seguridad que se levantan por sobre el mismo, depende de la persona que esté atrás y que va configurando. Con el venir de SDN se viene una revolución en todas las redes, donde todos los paradigmas que uno tiene hoy en día a nivel de redes, se van a romper porque es una manera diferente de pensar. 
